\title{
Anion Photoelectron Imaging Spectroscopy of Glyoxal
}

\author{
Tian Xue, Andrew R. Dixon, and Andrei Sanov* \\ Department of Chemistry and Biochemistry, The University of Arizona, Tucson, AZ 85721, \\ U.S.A. \\ *Email:sanov@u.arizona.edu
}

We report a photoelectron imaging study of the radical-anion of glyoxal. The $532 \mathrm{~nm}$ photoelectron spectrum provides the first direct spectroscopic determination of the adiabatic electron affinity of glyoxal, EA $=1.10 \pm 0.02 \mathrm{eV}$. This assignment is supported by a FranckCondon simulation of the experimental spectrum that successfully reproduces the observed spectral features. The vertical detachment energy of the radical-anion is determined as VDE $=$ $1.30 \pm 0.04 \mathrm{eV}$. The reported EA and VDE values are attributed to the most stable $\left(C_{2 h}\right.$ symmetry) isomers of the neutral and the anion. 


\section{Introduction}

Small dicarbonyls play important roles in atmospheric chemistry. The simplest of these molecules is glyoxal (OHCCHO), shown in Figure 1 (top left). Although it is a minor component of the atmosphere, its contributions to the chemistry of volatile organic compounds [1-4] make it important to the modeling of atmospheric processes. Specifically, glyoxal and its derivatives can be oxidized, hydrolyzed, and oligomerized through heterogeneous reactions to form dimers and larger complexes [5-7]. The oligomers in particular are known to be a source of oxidizing radicals and secondary organic aerosols (SOA) [8-10]. Although the effect of glyoxal oligomers on the modeling of SOAs has not been quantified, laboratory experiments suggest their importance [11]. Perhaps most significantly, glyoxal and other dicarbonyls can form lightabsorbing imidazoles, photosensitizing the growth of organic aerosols [12-15]. In addition, very recently glyoxal has emerged as a precursor for several small reactive intermediates with interesting properties, such as the OCCO diradical [16] and the OHCCO and HOCCO radicals [17]. It is in fact these species that motivated our initial interest in the anionic chemistry of glyoxal in general, and the glyoxal radical-anion in particular.

Despite its importance, some of the most fundamental properties of the glyoxal molecule and its anion remain unknown or poorly defined. In particular, the electron affinity (EA) of glyoxal has not been measured. The value appearing in contemporary databases, $\mathrm{EA}=0.62 \pm$ $0.26 \mathrm{eV}$ [18], is an indirect estimate determined from the enthalpies of formation of the anion and the neutral in a study of vinylene carbonate [11]. This indirect result is inconsistent with the Gaussian-3 (G3MP2B3) method predicting ca. $1.0 \mathrm{eV}$ [18].

The photochemistry of neutral glyoxal has received more attention. It was studied using fluorescence and UV-visible spectroscopy, while computational studies have focused on 
dimerization [19], dissociation [20] and hydrolysis [21]. Laser-induced fluorescence (LIF) measurements located the lowest-excited singlet state $2.728 \mathrm{eV}$ above the ground state [22]. The lowest triplet state was also observed just below the singlet transition, at $2.381 \mathrm{eV}[23]$ and the singlet-triplet coupling has been studied using level-anti-crossing spectroscopy [24-26]. Of particular interest to atmospheric chemistry is glyoxal unimolecular dissociation $[20,27]$. In one of the known channels, photoexcited $\mathrm{OHCCHO}$ forms two $\mathrm{CHO}$ radicals [28], while most famous is the "triple whammy" dissociation into two $\mathrm{CO}$ molecules and $\mathrm{H}_{2}$ [29].

Theoretical evidence from the previous as well as present work clearly shows that the $\mathrm{C}_{2 h}$ symmetry isomer (Figure 1) corresponds to the global minima of both the neutral and anion species $[30,31]$. Therefore, from this point on, whenever we refer simply to "glyoxal", without specifying the isomer, the $C_{2 h}$ species is implied. The height of the internal rotation barrier between the $C_{2 h}$ and $C_{2 v}$ neutral isomers was investigated experimentally, with the results

varying from $1770 \pm 60 \mathrm{~cm}^{-1}$ by from During, Bucy and Cole in 1975 [32] to $2077 \mathrm{~cm}^{-1}$ by Butz in 1990 [27] (all values are with respect to the $\mathrm{C}_{2 h}$ minimum). Theoretical predictions of the barrier height vary from $1960 \mathrm{~cm}^{-1}$, as determined by Scuseria and Schaefer in 1989 [30], to $2400 \mathrm{~cm}^{-1}$ by Kakumoto in 1987 [31].

We report the first direct experimental determination of the electron affinity of glyoxal and the vertical detachment energy (VDE) of the radical-anion of glyoxal using anion photoelectron imaging spectroscopy. The experimental results, supported by additional theoretical calculations and Franck-Condon simulations, provide new insight into the electronic structure of the glyoxal molecule and its radical-anion.

\section{Experimental and Theoretical Methods}

The experiments were performed using a custom-built anion photoelectron imaging spec- 
trometer described previously [33,34]. The glyoxal solution (Glyoxal, pure, $40 \% \mathrm{wt} / \mathrm{wt}$ solution in water, ACROS Organics) was first partially dehydrated using a $\sim 1: 1$ volume ratio of $3 \AA$ molecular sieves for at least 24 hours until the solution was yellowish rather than colorless and transparent. The solution was then extracted from the sieve mixture using methanol [35]. The resulting solution was heated to $70-85{ }^{\circ} \mathrm{C}$ in order to raise its vapor pressure. The vapor was seeded into an $\mathrm{N}_{2} \mathrm{O}$ carrier gas with a backing pressure of 20 psi. The mixture was expanded into the source chamber through a pulsed supersonic nozzle (General Valve, Inc., Series 9) operated at a repetition rate of $50 \mathrm{~Hz}$. The expansion was crossed with a collimated beam of 1 $\mathrm{keV}$ electrons from an electron gun. Anions were formed by attachment of slow secondary electrons in the electron-impact ionized plasma [35].

Glyoxal anions were interrogated by linearly polarized laser pulses at $532 \mathrm{~nm}$ obtained by frequency doubling the fundamental output a Spectra Physics LAB-130-50 Nd:YAG laser. The laser pulses were timed to interact only with the ions of a mass-to-charge ratio $\mathrm{m} / \mathrm{z}=58$. Photoelectrons were velocity-mapped [36] in the direction perpendicular to the ion and laser beams and projected onto a $40 \mathrm{~mm}$ diameter dual microchannel-plate detector, coupled to a P43 phosphor screen. Photoelectron impact positions were recorded by a thermoelectrically cooled charge-coupled-device camera. Images were typically accumulated for $\sim 10^{6}$ experimental cycles. The complete three-dimensional photoelectron distribution was reconstructed via an inverse Abel transformation [37] implemented in the BASEX program [38]. The resulting radial distributions were converted to photoelectron spectra using the well-known $\mathrm{O}^{-}$photodetachment transitions for calibration $[39,40]$.

Electronic structure calculations and geometry optimizations were performed using the GAUSSIAN 09 software package [41]. Geometry optimizations using the coupled-cluster 
method with single and double excitations (CCSD) with Dunning's augmented correlationconsistent basis of triple- $\zeta$ quality (aug-cc-pVTZ) were performed for both the anion and neutral species of glyoxal, yielding both the $C_{2 h}$ and $C_{2 v}$ symmetry equilibrium structures. These were used in the calculations of adiabatic electron affinities (EA) and vertical detachment energies (VDE). The EAs were calculated as the difference in the electronic energy for the glyoxal anion and neutral species at the respective optimized geometries $\left(C_{2 h}\right.$ or $\left.C_{2 v}\right)$. The VDEs were calculated as the energy difference for the anion and the neutral species, both at the optimized geometry of the anion. Zero-point vibrational energy corrections were determined using singlepoint harmonic frequency calculations at the same level of theory but with the smaller aug-ccpVDZ basis set, after re-optimizing the structures with the smaller basis.

The CCSD/aug-cc-pVTZ optimized geometries and CCSD/aug-cc-pVDZ vibrational frequencies were also used for the normal mode analysis as part of the Franck-Condon (FC) simulations. These simulations were carried out using the PESCAL 2010 software, with the normal modes treated as uncoupled harmonic oscillators with full Duschinsky rotation using the Chen method $[42,43]$.

\section{Results and Analysis}

The raw photoelectron image of glyoxal anion collected at $532 \mathrm{~nm}$ is shown in Figure 2 along with the corresponding spectrum. The spectrum is plotted versus electron binding energy: $\mathrm{eBE}=h v-\mathrm{eKE}$, where $h v$ is the photon energy and eKE is the kinetic energy of the photoelectrons. The image indicates slightly negative photoelectron anisotropy with respect to the laser polarization direction, along with some limited vibrational resolution discernable in the spectrum.

The spectrum consists of the main intense band labeled A with partial vibrational resolu- 
tion and a weak shoulder (B) extending from under the major feature A towards lower eBEs. Later in this Section, band A will be assigned to the $X^{1} \mathrm{~A}_{g} \leftarrow X^{2} \mathrm{~A}_{u}$ photodetachment transition in the radical-anion of glyoxal $\left(C_{2 h}\right.$ isomer). The anion VDE is defined approximately by the position of the overall band A maximum. It was determined as $\mathrm{VDE}=1.30 \pm 0.04 \mathrm{eV}$ by fitting a broad Gaussian envelope to the entire band.

The maximum of the first peak (marked ' 0 ' in Figure 2) corresponds to the adiabatic EA of glyoxal, determined as $\mathrm{EA}=1.10 \pm 0.02 \mathrm{eV}$. This directly determined value is significantly different from the previous indirectly obtained result $\mathrm{EA}=0.62 \pm 26 \mathrm{eV}$ [11], presently cited in databases [18]. On the other hand, the new spectroscopic determination is in a much better agreement with the G3MP2B3 theory prediction of EA $\approx 1.0 \mathrm{eV}[18]$.

To aid in the detailed analysis of the photoelectron spectrum, the geometries of both the neutral and anion species of glyoxal were optimized at the CCSD/aug-cc-pVTZ level, for both the $C_{2 v}$ and $C_{2 h}$ symmetry isomers. The optimized geometric parameters are included in Figure 1 (in bold for the anion and in italics for the neutral). The calculated EAs and VDEs of the respective neutral and anion species are summarized in Table 1 . The position of the 0 peak in the experimental spectrum (Figure 2) matches well with the $C_{2 v}$ EA value, but the experimental VDE is a better match for the corresponding $C_{2 h}$ prediction. Clearly, additional analysis is required for a consistent interpretation of the data.

For both the anion and the neutral, the $C_{2 h}$ structures are more stable than their $\mathrm{C}_{2 v}$ counterparts. Indeed, the previous studies established that the most stable structure of neutral glyoxal corresponds to the $C_{2 h}$ isomer (Figure 1, left), with a $C_{2 h}$ to $C_{2 v}$ internal rotation (isomerization) barrier of $2077 \mathrm{~cm}^{-1}$ [27]. This barrier height is compared to the $1688 \mathrm{~cm}^{-1}$ energy difference between the two isomers, determined by dispersed fluorescence spectroscopy [27] (or $1597 \mathrm{~cm}^{-1}$ 
based on our calculations summarized in Figure 1). The corresponding barrier height on the anion potential energy surface is not known. However, the electronic structure calculations described above indicate that the additional electron in the anion occupies an $a_{u}$ molecular orbital, which has a bonding $\pi$ character with respect to the $\mathrm{C}-\mathrm{C}$ bond. As a consequence, there is noticeable shortening of the $\mathrm{C}-\mathrm{C}$ bond from neutral glyoxal to the anion (see Figure 1). Given the additional (relative to the neutral) $\pi$ bonding character, we infer that the internal-rotation barrier with respect to the $\mathrm{C}-\mathrm{C}$ bond on the anion potential must be higher than that on the neutral. Therefore, the $C_{2 h}$ to $C_{2 v}$ isomerization should be less likely in the anion, compared to the neutral, and hence the dominant isomer of the anions generated by electron attachment to $C_{2 h}$ glyoxal molecules should follow the same $C_{2 h}$ structural motif.

The photoelectron spectrum expected in the photodetachment of the $C_{2 h}$ anion was simulated using the PESCAL program $[42,43]$. The energy of the $0-0$ photodetachment transition was set to $1.10 \mathrm{eV}$, matching peak 0 in Figure 2 and the starting ab initio anion geometry shown in Figure 1 (left) was adjusted to match the experimental spectrum (band A). The adjustment was done by treating the displacements along the neutral normal modes as adjustable parameters in order to match the final FC simulation to the experimental spectrum. In the process, the calculated FC intensities were multiplied by an $\mathrm{eKE}^{3 / 2}$ pre-factor, accounting for the Wigner-like [44] scaling of the electronic part of the photodetachment cross-section [45]. The scaled FC stick spectrum is included in Figure 2, while the adjusted geometric parameters of the anion are included in Figure 1 (left) in plain font for direct comparison to the ab initio predictions in bold.

The stick spectrum was convoluted with an instrumental resolution function. The convolution was carried out in the speed domain and the resolution function was taken to be a Gaussian 
of a $\mathrm{FWHM}=2 \times 10^{4} \mathrm{~m} / \mathrm{s}$, as determined from $\mathrm{O}^{-}$photodetachment under similar experimental conditions. The convoluted spectrum was then transformed into the eBE domain using the appropriate Jacobian. The result is shown in Figure 2 in purple, overlaid with the experimental spectrum.

The agreement between the FC simulation and the experimental spectrum leaves little doubt about the robustness of the adjusted geometric parameters of the glyoxal anion included in Figure 1. A similar simulation was attempted for the $C_{2 v}$ anion isomer, but it failed to reproduce the observed spectral features, providing additional confirmation that the data in Figure 2 (band A) do indeed correspond to the $C_{2 h}$ isomer of the anion.

The minor feature, band B appearing as a broad low-eBE tail in the spectrum, cannot be accounted for by one-photon detachment of the glyoxal anion. Possible explanations for it are, therefore: hot bands, a two-photon process, or a coincident mass-to-charge species. The feature extending more than half an electron-volt below the adiabatic EA is unlikely to be a hot band. A two-photon process is possible, but the signal intensity was insufficient to determine band A's scaling with laser power.

In the absence of conclusive proof, the most plausible explanation seems to be that band B corresponds to a coincident species with the same mass-to-charge ratio as the glyoxal anion, $\mathrm{m} / \mathrm{z}$ $=58$. The most likely candidate is the solvated anion of methylene, $\mathrm{CH}_{2}{ }^{-} \cdot \mathrm{N}_{2} \mathrm{O}$. In preparation of the glyoxal sample, the dehydrated glyoxal solution was extracted from the sieve mixture using methanol, as described in Section 2. The methylene anion could be produced in our ion source from the residual methanol present in the sample. Supporting this hypothesis, an $m / z=31$ species, corresponding to the methoxide anion $\left(\mathrm{CH}_{3} \mathrm{O}^{-}\right)$, was also observed in the primary ion mass-spectrum, as was $m / z=75\left(\mathrm{CH}_{3} \mathrm{O}^{-} \cdot \mathrm{N}_{2} \mathrm{O}\right)$. The electron affinity of $\mathrm{CH}_{2}$ is $0.652 \mathrm{eV}$ [46], 
while the solvation energy by $\mathrm{N}_{2} \mathrm{O}$ is expected to be $\sim 0.2 \mathrm{eV}$, making the $\mathrm{CH}_{2}^{-} \cdot \mathrm{N}_{2} \mathrm{O}$ cluster anion, whose mass is nominally coincident with that of glyoxal, a viable candidate for the origin of the weak B band observed in Figure 2.

\section{Summary}

Using photoelectron imaging spectroscopy, we have measured the photoelectron spectrum of the glyoxal anion and obtained the first direct spectroscopic determination of the adiabatic electron affinity of glyoxal, EA $=1.10 \pm 0.02 \mathrm{eV}$. The vertical detachment energy of the radicalanion of glyoxal is determined as $\mathrm{VDE}=1.30 \pm 0.04 \mathrm{eV}$. These values are attributed to the most stable $\left(C_{2 h}\right)$ isomers of the neutral and the anion. Franck-Condon simulations indicate several vibrational modes activated upon electron photodetachment and successfully reproduce the observed spectral features. Future studies will examine the effects of substituents on the electron-binding properties of the molecule.

\section{Acknowledgements}

We thank Dr. Dmitry Khuseynov for his encouragement to use glyoxal as the precursor in this work. William P. Brezinski and Dr. Richard F. Vreeland are acknowledged for their help with dehydration of the precursor. This work was supported by the U.S. National Science Foundation (grant CHE-1266152). 


\section{References}

[1] J. P. DiGangi, et al., Atmos. Chem. Phys. 12, 9529 (2012).

[2] K. F. Ho, S. S. H. Ho, W. T. Dai, J. J. Cao, R. J. Huang, L. W. Tian, W. J. Deng, Environ. Monit. Assess. 186, 2835 (2014).

[3] C. J. Kampf, et al., Atmos. Chem. Phys. 12, 6145 (2012).

[4] M. Vrekoussis, F. Wittrock, A. Richter, J. P. Burrows, Atmos. Chem. Phys. 9, 4485 (2009).

[5] H. E. Krizner, D. O. De Haan, J. Kua, J. Phys. Chem. A 113, 6994 (2009).

[6] D. O. De Haan, A. L. Corrigan, M. A. Tolbert, J. L. Jimenez, S. E. Wood, J. J. Turley, Environ. Sci. Technol. 43, 8184 (2009).

[7] R. Zhao, A. K. Y. Lee, J. P. D. Abbatt, J. Phys. Chem. A 116, 6253 (2012).

[8] B. M. Connelly, D. O. De Haan, M. A. Tolbert, J. Phys. Chem. A 116, 6180 (2012).

[9] A. G. Carlton, B. J. Turpin, K. E. Altieri, S. Seitzinger, A. Reff, H. J. Lim, B. Ervens, Atmos. Environ. 41, 7588 (2007).

[10] M. M. Galloway, M. H. Powelson, N. Sedehi, S. E. Wood, K. D. Millage, J. A. Kononenko, A. D. Rynaski, D. O. De Haan, Environ. Sci. Technol. 48, 14417 (2014).

[11] R. N. Compton, P. W. Reinhardt, H. C. Schweinler, Int. J. Mass Spectrom. 49, 113 (1983).

[12] S. Rossignol, K. Z. Aregahegn, L. Tinel, L. Fine, B. Noziere, C. George, Environ. Sci. Technol. 48, 3218 (2014).

[13] D. O. De Haan, L. N. Hawkins, J. A. Kononenko, J. J. Turley, A. L. Corrigan, M. A. Tolbert, J. L. Jimenez, Environ. Sci. Technol. 45, 984 (2011).

[14] N. Sedehi, H. Takano, V. A. Blasic, K. A. Sullivan, D. O. De Haan, Atmos. Environ. 77, 656 (2013).

[15] G. T. Drozd, V. F. McNeill, Environ. Sci.-Process Impacts 16, 741 (2014).

[16] A. R. Dixon, T. Xue, A. Sanov, Angew. Chem. Int. Ed. 54, 8764 (2015). 
[17] A. R. Dixon, T. Xue, A. Sanov, J. Chem. Phys. (in press).

[18] J. E. Bartmess, "Negative Ion Energetics Data" in NIST Chemistry WebBook, NIST Standard Reference Database Number 69, Eds. P. J. Linstrom, W. G. Mallard, National Institute of Standards and Technology, Gaithersburg MD, 20899, http://webbook.nist.gov (retrieved June 3, 2016).

[19] J. Kua, S. W. Hanley, D. O. De Haan, J. Phys. Chem. A 112, 66 (2008).

[20] D. M. Koch, N. H. Khieu, G. H. Peslherbe, J. Phys. Chem. A 105, 3598 (2001).

[21] M. K. Hazra, J. S. Francisco, A. Sinha, J. Phys. Chem. A 118, 4095 (2014).

[22] M. Gurnick, J. Chaiken, T. Benson, J. D. McDonald, J. Chem. Phys. 74, 99 (1981).

[23] L. H. Spangler, D. W. Pratt, J. Chem. Phys. 84, 4789 (1986).

[24] E. P. Peyroula, R. Jost, M. Lombardi, P. Dupre, Chem. Phys. 102, 417 (1986).

[25] E. Pebaypeyroula, R. Jost, M. Lombardi, J. P. Pique, Chem. Phys. 106, 243 (1986).

[26] P. Dupre, R. Jost, M. Lombardi, Chem. Phys. 91, 355 (1984).

[27] K. W. Butz, D. J. Krajnovich, C. S. Parmenter, J. Chem. Phys. 93, 1557 (1990).

[28] R. J. Salter, M. A. Blitz, D. E. Heard, M. J. Pilling, P. W. Seakins, J. Phys. Chem. A 113, 8278 (2009).

[29] L. M. Dobeck, H. M. Lambert, W. Kong, P. J. Pisano, P. L. Houston, J. Phys. Chem. A 103, 10312 (1999).

[30] G. E. Scuseria, H. F. Schaefer, J. Am. Chem. Soc. 111, 7761 (1989).

[31] K. Saito, T. Kakumoto, I. Murakami, J. Phys. Chem. 88, 1182 (1984).

[32] J. R. Durig, W. E. Bucy, A. R. H. Cole, Canadian Journal of Physics 53, 1832 (1975).

[33] L. Velarde, T. Habteyes, A. Sanov, J. Chem. Phys. 125, 114303 (2006).

[34] A. Sanov, R. Mabbs, Int. Rev. Phys. Chem. 27, 53 (2008). 
[35] M. A. Johnson, W. C. Lineberger, "Pulsed Methods for Cluster Ion Spectroscopy" in Techniques for the Study of Ion Molecule Reactions, Eds. J. M. Farrar, W. H. Saunders, Wiley, New York, 1988, p. 591-635.

[36] A. T. J. B. Eppink, D. H. Parker, Rev. Sci. Instrum. 68, 3477 (1997).

[37] A. J. R. Heck, D. W. Chandler, Annu. Rev. Phys. Chem. 46, 335 (1995).

[38] V. Dribinski, A. Ossadtchi, V. A. Mandelshtam, H. Reisler, Rev. Sci. Instrum. 73, 2634 (2002).

[39] D. M. Neumark, K. R. Lykke, T. Andersen, W. C. Lineberger, Phys. Rev. A 32, 1890 (1985).

[40] S. J. Cavanagh, S. T. Gibson, M. N. Gale, C. J. Dedman, E. H. Roberts, B. R. Lewis, Phys. Rev. A 76, 052708 (2007).

[41] M. J. Frisch, et al., Gaussian 09. Gaussian, Inc., Wallingford, CT, 2009.

[42] K. M. Ervin, T. M. Ramond, G. E. Davico, R. L. Schwartz, S. M. Casey, W. C. Lineberger, J. Phys. Chem. A 105, 10822 (2001).

[43] P. Chen, Unimolecular and Bimolecular Reactions Dynamics, Eds. C.-Y. Ng, T. Baer, I. Powis, John Wiley \& Sons, Chichester, 1994, p. 371.

[44] E. P. Wigner, Phys. Rev. 73, 1002 (1948).

[45] D. Khuseynov, A. R. Dixon, D. J. Goebbert, A. Sanov, J. Phys. Chem. A 117, 10681 (2013).

[46] D. G. Leopold, K. K. Murray, A. E. S. Miller, W. C. Lineberger, J. Chem. Phys. 83, 4849 (1985). 
Table 1. The EA and VDE values calculated with the CCSD/aug-cc-pVTZ methodology for glyoxal (anion), compared to the experimental determinations. The calculated EA values are listed with and without the zero-point vibrational energy (ZPE) corrections. The ZPE corrections were determined at the same level of theory, but with the aug-cc-pVDZ basis set.

\begin{tabular}{ccccc}
\hline Isomer & Transition & \multicolumn{2}{c}{ EA / eV } & VDE / eV \\
& & w/o ZPE & with ZPE & \\
\hline$C_{2 h}$ & $X^{1} \mathrm{~A}_{g} \leftarrow X^{2} \mathrm{~A}_{u}$ & 0.935 & 0.976 & 1.256 \\
$C_{2 v}$ & $X^{1} \mathrm{~A}_{1} \leftarrow X^{2} \mathrm{~B}_{1}$ & 1.061 & 1.091 & 1.450 \\
\hline Experiment & & $1.10 \pm 0.02$ & $1.30 \pm 0.04$
\end{tabular}




\section{Figure Captions}

Figure 1. The $C_{2 h}$ (top left) and $C_{2 v}$ (top right) structures of the neutral and anion species of glyoxal optimized at the CCSD/aug-cc-pVTZ level of theory. The optimized geometric parameters for the neutral and the anion are given in italics and bold, respectively. The $C_{2 h}$ anion parameters adjusted as part of the Franck-Condon simulation of the experimental spectrum are shown in plain type. The bottom part of the figure shows a schematic energy diagram. The minimum energies of the $C_{2 h}$ and $C_{2 v}$ anion and neutral isomers of glyoxal are indicated relative to the most stable anion structure $\left(C_{2 h}\right)$. The relative energies are determined from the CCSD/aug-cc-pVTZ calculations and include the CCSD/aug-cc-pVDZ zero-point vibrational energy corrections, as explained in the text. The approximate isomerization barrier height indicated schematically for the neutral surface represents a preponderance of the past experimental and theoretical results [27,30-32]. The corresponding barrier height for the anion is not known, but is expected to be greater than that for the neutral, due to the $\mathrm{C}-\mathrm{C} \pi$ bonding character of the excess electron.

Figure 2. The $532 \mathrm{~nm}$ photoelectron image and the corresponding photoelectron spectrum of the glyoxal anion. Vertical double arrow indicates the laser polarization direction. The experimental spectrum is superimposed with the Frank-Condon simulation obtained as described in the text. 
Figure 1

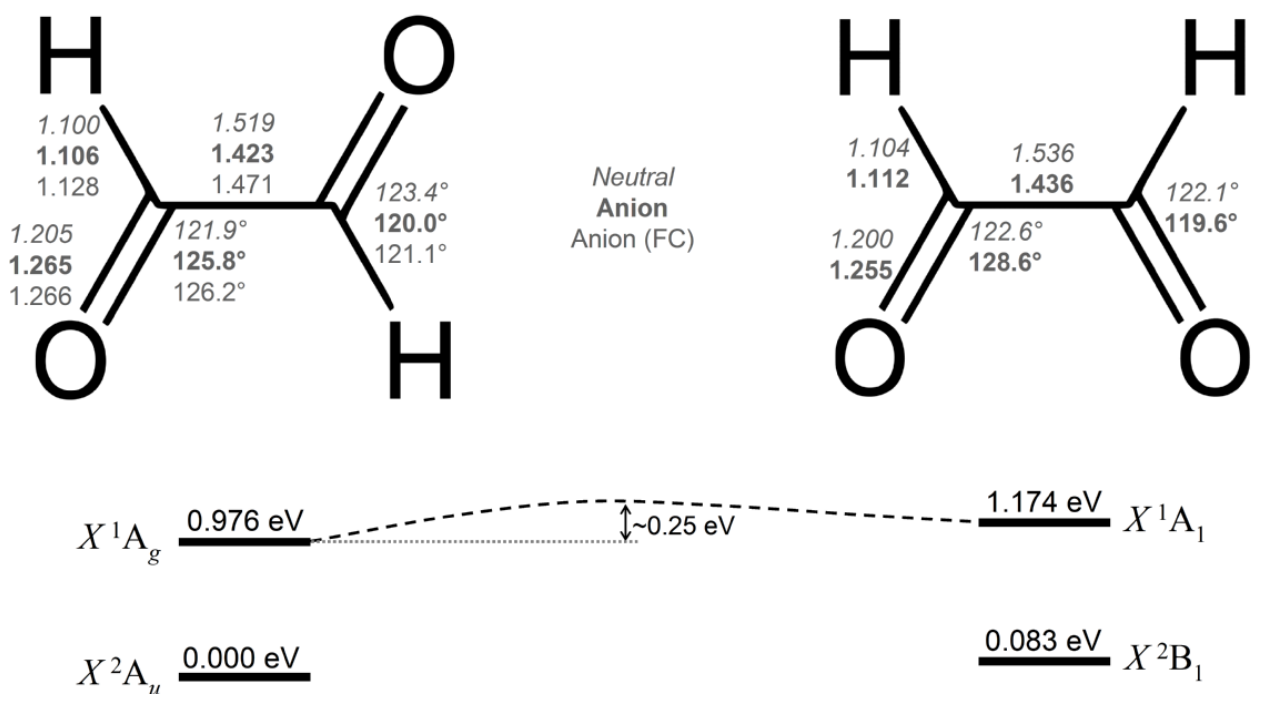


Figure 2

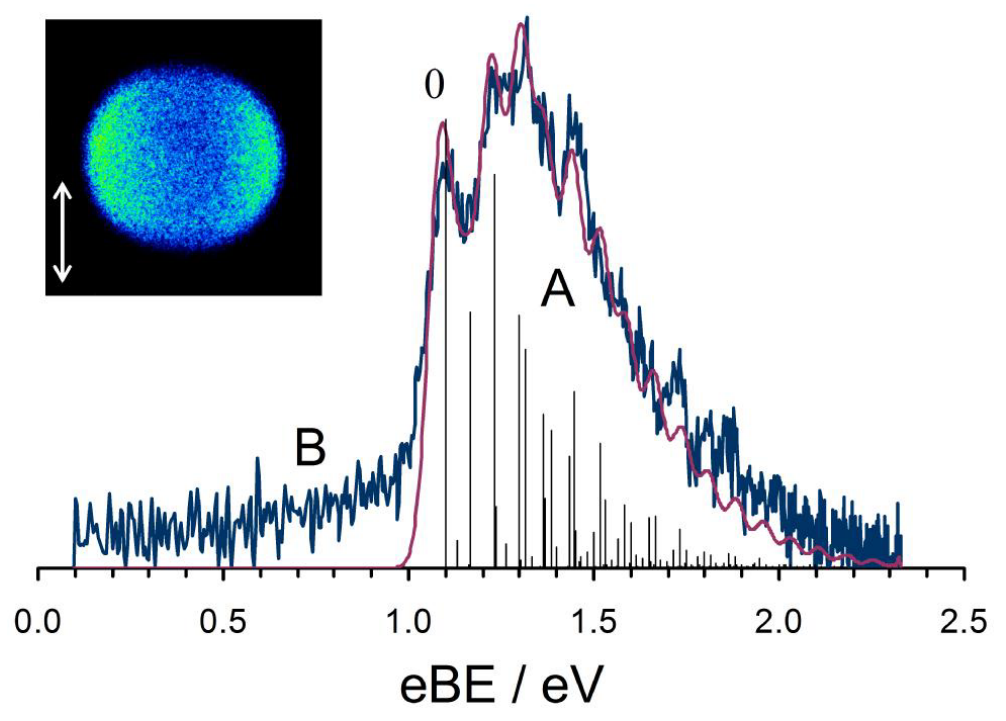




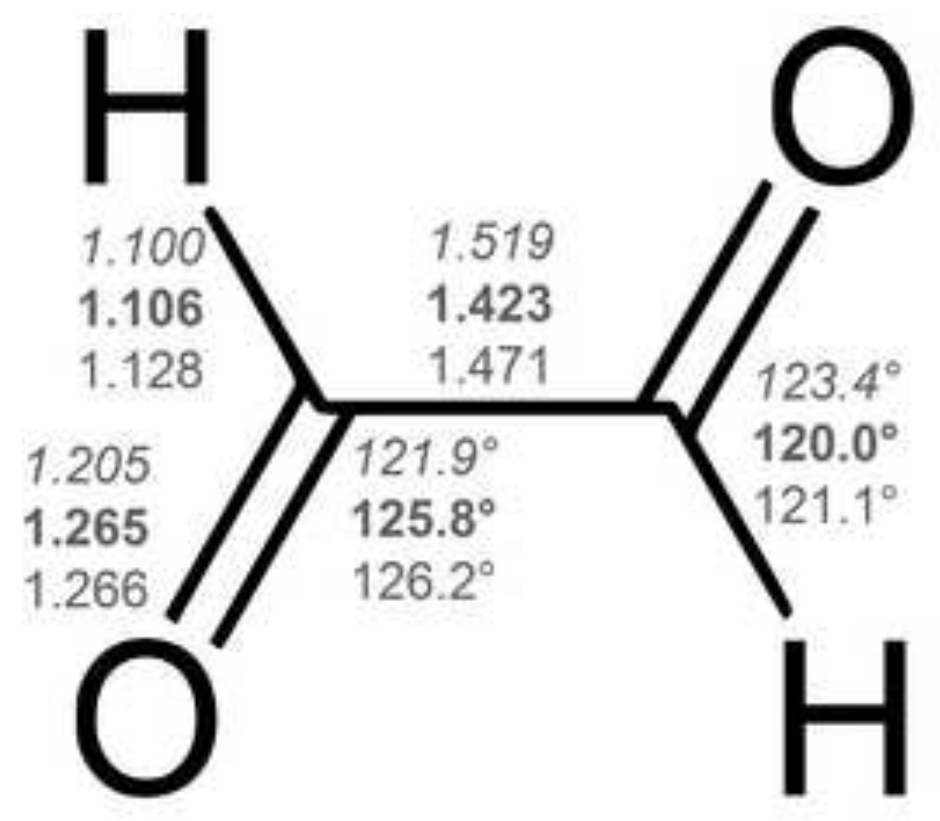

\section{Neutral \\ Anion \\ Anion (FC)}

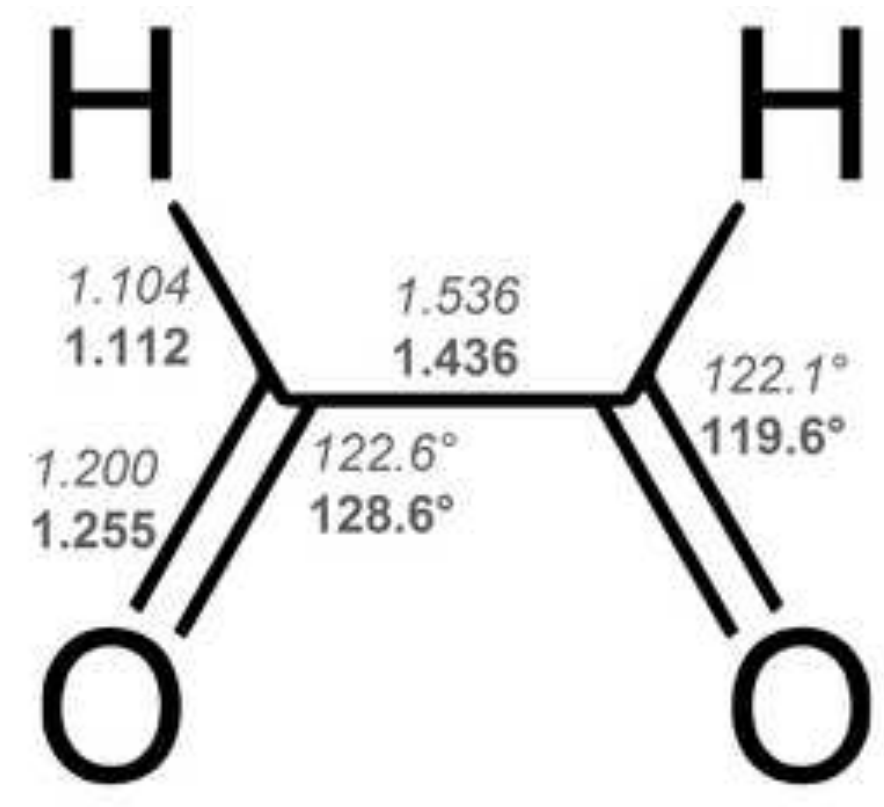

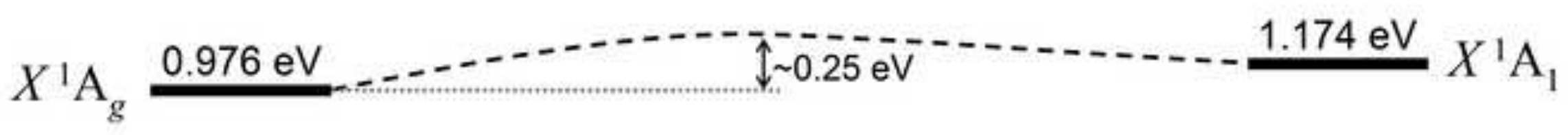

$X^{2} \mathrm{~A}_{u} \underline{0.000 \mathrm{eV}}$

$0.083 \mathrm{eV} X^{2} \mathrm{~B}_{1}$ 


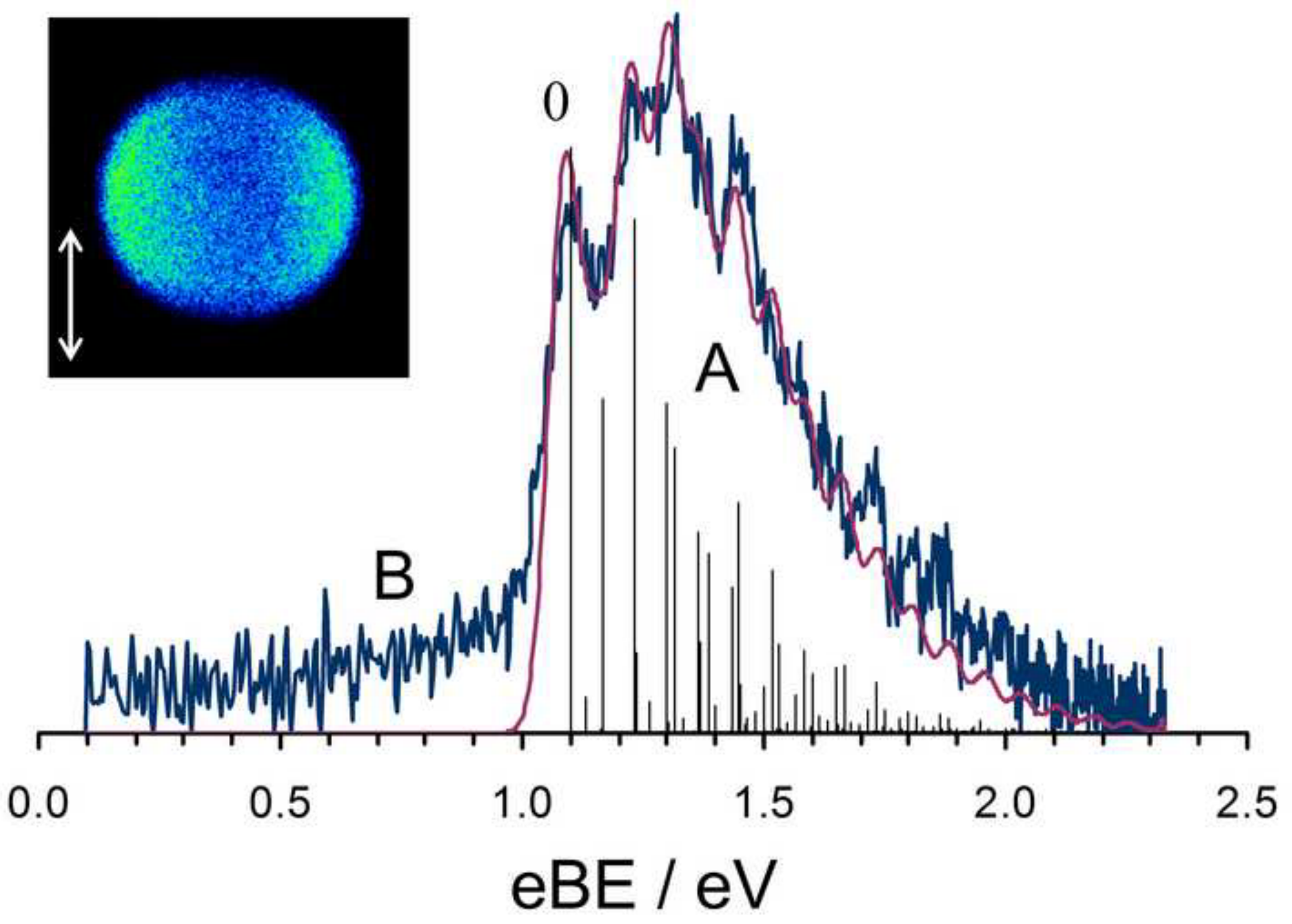



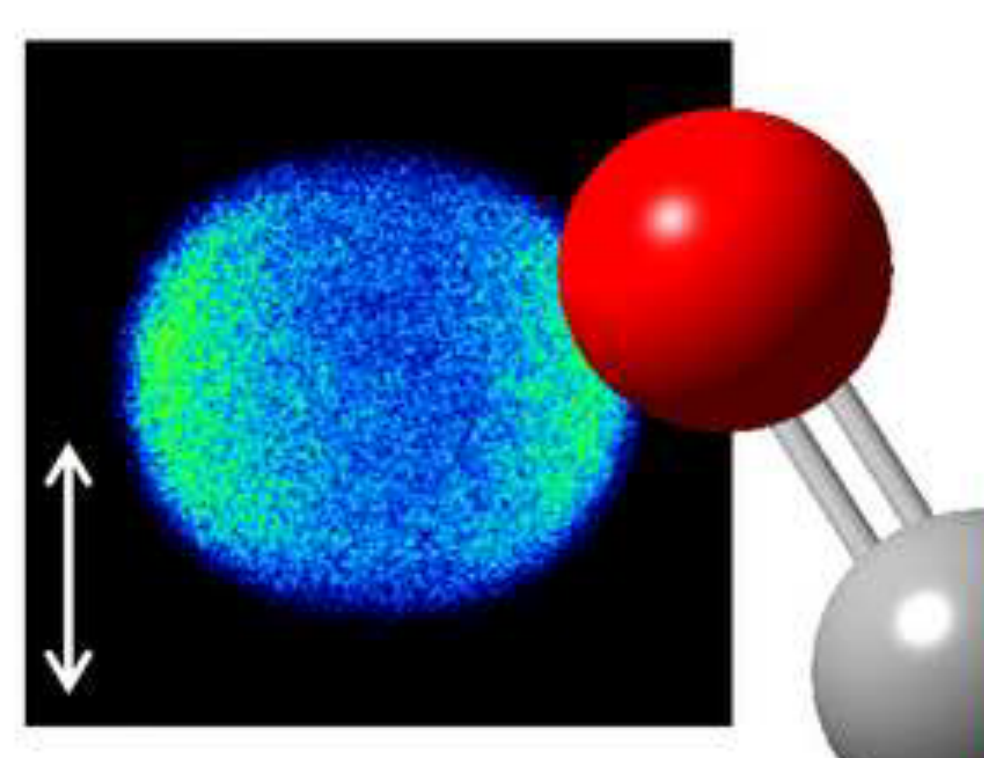

(1)
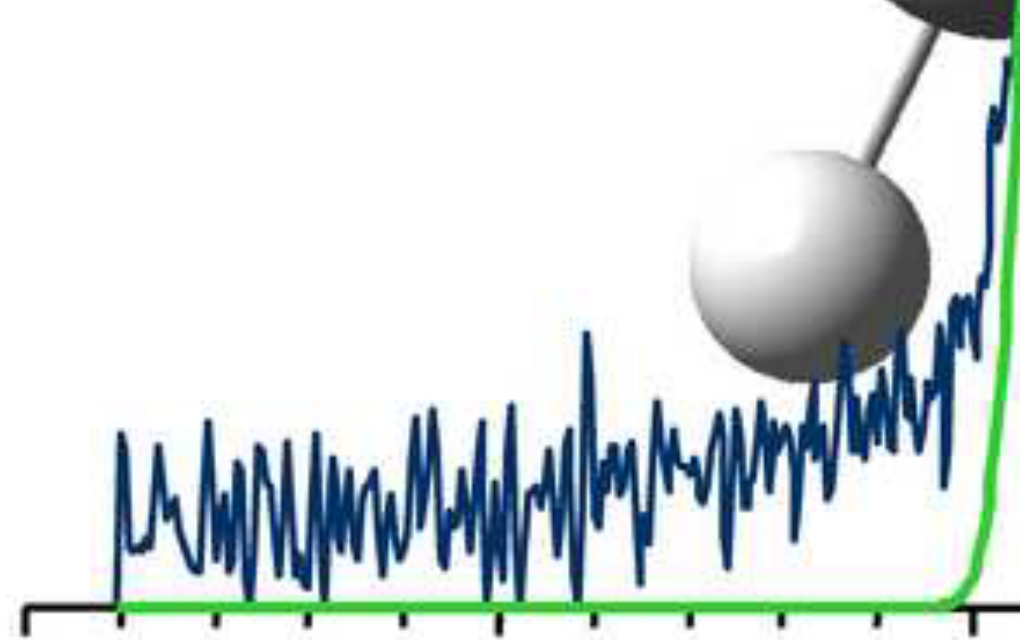

0.0

0.5

1.0

1.5

2.0

2.5

eBE / eV 